\title{
On-line Monitoring System Design of Electrical Equipment
}

\author{
Chenxi Zheng, Dong Zhao \\ North China Electric Power University, Peking 102206, China \\ 3079996990@qq.com
}

Keywords: intelligentization, remote monitoring, MODBUS fieldbus

\begin{abstract}
In nowadays' society, the informationization and intelligentization of the articles (e.g. household articles, articles of daily use, etc) has gradually walked into people's sight. To ensure the normal operation of the electrical equipment and the realization of the automatic processing under certain situation, the intelligent of this equipment has become more and more important. To establish on-line monitoring system for electrical equipment can provide operation platform for the automatic control of the future electrical equipment system. It receives the data of the distributor through RS-485 interface, and submits it to MODBUS bus through PLC. Then, the upper computer of a PC monitors the real-time parameters of each distribution. Through the industrial Ethernet, it is feasible to share the data achieved from this upper computer with other PCs for joint monitoring after authorization, so as to achieve the purpose of remote monitoring.
\end{abstract}

\section{Introduction}

With the development of the era, the electric power has played a vital role in the social life. In all industries, the normal work has become closer relationship with the electric power, and it cannot be separated from the electric power. To improve the power supply and distribution has profound significance for ensuring the normal development of all industries and realizing the national economic modernization.

Our power grid has obtained certain achievements in realizing the smart power grid construction for the remote and large-scale electric power transmission. Nowadays, the electrical equipment is developed towards the informationization and networking. To ensure the stable and safety operation of the power distribution system, it is necessary to collect and handle the work status of the electrical equipment. The achievement of this requirement is favorable for managing the coordinated operation and uniform management of each electrical equipment.

Through the analysis on the design proposal about the on-line monitoring system of the electrical equipment, this paper makes comment regarding the overall design of this system.

\section{Design proposal about the on-line monitoring system of the electric equipment}

Generally, the electric equipment and PLC (programmable logic device) are connected through the serial port communication, such as RS-485 interface. This design transmits the status information of the electric equipment to independent PLC through RS-485 interface. PLC can collects and transmits the data to an upper computer through MODBUS, and then realize the monitoring on electric equipment. Besides, it is feasible to share the status of the electric equipment with another upper computer, or authorize other equipment to handle and operate the status, etc.

This system adopts layered and distributed network structure, which is divided into field equipment layer, communication layer, and monitoring management layer.

(1) Field equipment layer. Through the arrangement of the physical addresses of the field equipment, it is feasible not only to review the physical address of the field equipment, but also to provide convenience for inquiring th ${ }^{1}$ e distribution circuit. The related electric equipment is equipped with RS-485 communication port. The electrical parameters of each power supply and distribution

\footnotetext{
${ }^{1}$ Supported by “the Fundamental Research Funds for the Central Universities”
} 
circuit can be monitored through the upper computer software. The upper computer and authorized monitoring equipment can handle and control the status of the loop and the report of the failure message.

(2) Communication layer. It provides data transmission channel for the electrical equipment, and transmits the collected status information of the equipment to other equipment and computers. This design adopts the serial MODBUS realized by RS-485 interface.

(3) Monitoring management layer. In this layer, one or more PCs monitor the status of the electric equipment in real time through the software, and also realize its data storage, alarming and prompt, failure recording, etc. To ensure the smooth communication, it is feasible to switch the uninterruptible batteries under electricity outage, with the purpose of ensuring the stable transmission of the data and preventing the data loss.

\section{Realization of the design proposal about the on-line monitoring system of the electric equipment}

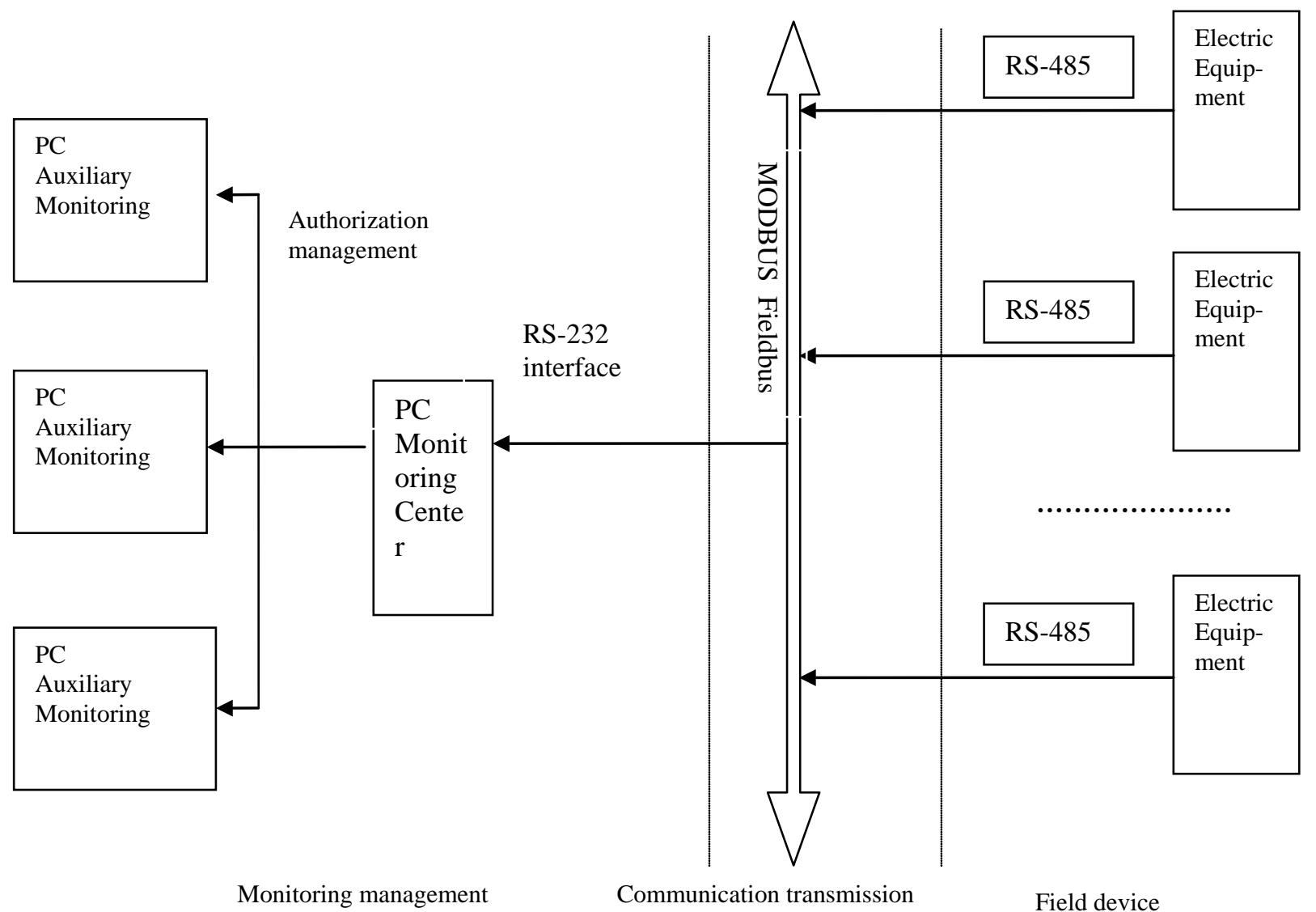

Fig.1 Structure Chart for On-line Monitoring System of Electric Equipment

As shown in Fig.1, this monitoring system design adopts a PC as the upper computer, which plays a role of monitoring the electric equipment. Meanwhile, it can send out specific instruction and change the equipment status, and then control the distribution circuit. PLC is connected with the electric equipment with RS-485 interface, and the upper computer is connected with MODBUS field bus through RS-232, so as to achieve the data transmission. This upper computer can be regarded as a data repeater. Its data can be shared through the industrial Ethernet. The PC after gaining the authorization can participate in monitoring and controlling the equipment.

In this design, the key to realize the function of the whole system includes two parts below: (1) the communication between the lower computer and MODBUS enables the lower computer to possess the communication capability and collect the status information of the electric equipment, and 
transmit it to the field bus; (2) the communication between the upper computer and MODBUS, and the database management of the upper computer.

\subsection{Communication between lower computer and MODBUS}

MODBUS is a common language applied in electronic control unit. Through this protocol, the controllers can be mutually communicated and it also can communicate with other equipment through the network (e.g. Ethernet). It has already become a common industrial standard. It enables the control equipment produced by different manufacturers to connect together and form the industrial network for centralized monitoring.

In MODBUS system, 2 transmission modes are optional. Each MODBUS system only can use a mode, no allowing for the mixing of 2 modes. One mode is ASCII (American Standard Code for Information Interchange), and the other one is RTU (remote terminal unit). To achieve higher density of the transmission data and faster transmission under the same Baud rate, RTU mode without the interval between the data frame during the transmission is selected. The mating data verification method is CRC check. CRC field has 2 bytes, including 16-digital binary value. It is added to the message after being calculated by the transmission equipment. Receive the CRC that gets the information recalculated by the equipment, and compare it with the received value in CRC field. There is some mistake if both values are different.

The electric equipment is connected with RS-485, and it transmits the data to MODBUS field bus through the interface for the upper computer to retrieve.

\subsection{Communication between the upper computer and MODBUS, and the database management of the upper computer}

The information transmission of MODUS under RTU mode is asynchronous mode, taking byte as the unit. The information frame format of the communication message transmitting between the master station and slave station is shown in Chart. 1.

The master-slave mode is selected. If the host equipment sends out a piece of information, slave equipment can return a response. Similarly, when slave equipment accepts the information, it will organize the response information of slave equipment, and return it to the original master equipment sending the information.

When the communication order is transmitted fro the master equipment to the slave equipment, the slave equipment conforming to the corresponding address code accepts the communication order, and reads the information according to the function codes and related requirements. If CRC check is correct, implement the corresponding tasks, and then return the implementation result to the master equipment.

\begin{tabular}{|c|c|c|c|}
\hline equipment address & function code & data content & CRC 16 check code \\
\hline 1 Byte & 1Byte & N Byte & 2 Byte \\
\hline \multicolumn{2}{|c|}{ PDU(Protocol Data Unit ) } \\
\hline \multicolumn{2}{|c|}{ ADU (Application Data Unit) } \\
\hline
\end{tabular}

Chart. 1 Information frame format of MODBUS under RTU mode

In this design, SQL database language is applied to compile the database. SQL is an operating command set especially established for the database. It is a database language with comprehensive function. When it is used, it is only necessary to give out the command of "what to do", and the users do not need to consider "how to do". SQL has great function. Besides, it is easy to learn and use. It already has become the foundation of the database operation. Moreover, almost all databases support SQL.

After successful construction, it is feasible to observe all parameters through the monitoring interface through designed UI interface, such as three-phase voltage, loop current I, active power P, reactive power $\mathrm{Q}$, apparent power $\mathrm{S}$, frequency f, power quality, etc. 


\section{Summary}

With the technology development, the whole society has showed higher requirements on the equipment automation and intelligentization. There are so many kinds of intelligent equipment. However, the power supply and distribution system in the electric system is the basis of all equipment with automation system.

The intelligent monitoring system of the equipment based on MODBUS field bus raised by this paper can realize the real-time and intelligent control on the electric equipment, and understand the work status of the equipment through simple UI interface. After the master device is successfully monitored, it is feasible to authorize controlling and monitoring other auxiliary monitoring PCs, and then ensure the safety operation of the electric equipment to the greatest extent.

\section{References}

[1]. Wang Li, Yang Li. Intelligent Power Distribution System Design and Realization Based on Modbus Bus, 2011.

[2]. Li Jiong. Design of Power Supply and Distribution System Based on PLC, 2011.

[3]. Xu Lufei. Design about On-line Monitoring System of Power Distribution Network, 2010. 